\title{
Link between Successful Aging and Selected Communication-demographic Characteristics of Filipino Elders
}

\author{
By Carmencita P. Del Villar
}

\begin{abstract}
This study looked into the possible correlation between successful aging and selected communication-demographic characteristics of Filipino elders. Over 800 elder respondents, ages 60 to 95, males and females, from different places of residence and socio-economic classes were invited as respondents in the survey. To complement the quantitative data from the survey, interviews and focus-group-discussions were also conducted. The selected 20 communication-demographic factors were each statistically tested to determine their possible correlation with successful aging. Results revealed that of the 20 factors tested, 12 were significantly linked to successful aging. The study concluded that Filipino elders would most likely experience successful aging if they were healthy, single, from the middle or upper socio-economic classes, with at least a college education, engaged in leisure activities on a regular basis, exercising regularly, with a number of close family members and friends and communicating with them often, and actively involved in religious activities. Their age, sex, occupation, place of residence, continuing education, mode of communication with close family and friends, type of religion, and commitment to continuing education were not significantly linked to successful aging.
\end{abstract}

Keywords: Successful aging, Filipino elders, communication-demographic factors.

\section{Introduction}

This study looked into the possible link between successful aging and selected communication-demographic factors among Filipinos. Twenty factors (age, sex, health, occupation, place of residence, civil status, socio-economic status, educational level, continuing education or training, leisure activities, frequency of leisure activities, exercise regimen, number of close family members, frequency of communication with close family members, mode of communication with close family members, number of close friends, frequency of communication with close friends, mode of communication with close friends, religion, and status in religious affiliation) were each statistically tested to determine their possible correlation with successful aging. This research is an extension of the Del Villar 2015 study which determined the correlation between Filipino elders' successful aging and selected cultural orientations. Results from the 2015 study showed that cultural orientations of power distance, collectivism, masculinity, and subjectivity to nature were significantly related to Filipino elders' experience of successful aging. With the 2015 findings, the present study sought to further

*Professor, University of the Philippines, Philippines.

https://doi.org/10.30958/ajss.5-3-3

doi=10.30958/ajss.5-3-3 
evaluate the same 816 respondents by focusing on their communicationdemographic profile and its possible link to successful aging.

\section{Study Frameworks}

The following sections describe the important concepts that guided the present study. These are successful aging and the importance of communicationdemographic factors in the successful aging of elders.

\section{Successful Aging Defined}

From the 2015 Del Villar study, the following definition of successful aging was conceptualized. It is defined as the combination of important elements that make elders reach their optimum best. These are: A positive attitude about aging, the right coping strategies to deal with the physical and emotional changes that inevitably come with the aging process, a healthy self-worth, and a generally happy disposition. It is believed that, with the right attitude, coupled with proactive and meaning-based strategies, a positive self-esteem, and the ability to see the happy side of life, one would be able to effectively conquer the challenges of aging.

The four elements that make up successful aging are discussed below:

\section{Attitudes about Aging}

Wrench, McCroskey, \& Richmond (2008) define attitude as "a predisposition.... a tendency that we have to do something...a tendency to evaluate people, ideas, or objects. Evaluative... means making judgments of good or bad, desirable or undesirable, or likable or unlikable (142). "It is a "hypothetical construct" which means that, similar to belief and value, it cannot be perceived by the senses and exists only in the mind. Although it exists only in the mind, it influences our behavior. Our evaluation of something determines how we behave toward it.

One's attitude about aging is important in the sense that it has been found to be associated with life satisfaction. According to Elejalde-Ruiz (2011), attitude can make all the difference in that how one felt and believed could actually influence one's health and well-being. In an experiment conducted by psychologist Langer (1979 as cited in Elejalde-Ruiz 2011), it was concluded that it was one's belief that lead elders to set restrictions or non-restrictions on themselves. If they believed they were healthy and would live long then they would. Their belief was more important than biology. Sheehy (1995), too, concluded that "it is our psychological attitude and behavior that more likely determine the quality and duration of our third age (419)" and not just our genes.

Levy (1975, as cited in Beck 2009), a social psychologist, reported that those who held negative attitudes towards aging were significantly more likely to have old age diseases when they grew old compared to those who held 
positive attitudes. Further, those with positive attitudes lived longer by as much as 7.5 years than those with negative views. An explanation provided was that of the concept of self-fulfilling prophecies where "those who believe that older people remain active, vital and healthy members of society may take better care of themselves, continuing good eating and exercise patterns. They can see a point in giving up smoking or beginning an exercise plan even in their 60s and 70s. Conversely, people who thought that aging inevitably brought infirmity and illness could consciously or unconsciously let that happen (10)."

Heckhausen \& Krueger (1993) concluded that the older the adults, the more positive their attitudes tended to be. Heckhausen \& Brim (1997) further found that the older adults' problems were consistent with their belief.

Cohen (2005) reported that older adults experienced "high morale... even among those who are frail. The positive outlook of people ... isn't the exception it's the rule (14)." Cohen forwarded a number of factors responsible for this positive disposition among which were: "greater acceptance of life's realities, a greater sense of self, and a long-term perspective that makes it easier to accept the inevitable slings and arrows of daily life". Cohen further explained that in addition to those psychological factors, new research proved that "changes in the older brain itself play an important role in the emotional aplomb and equanimity of many older adult (14)." Older brains, because of the physiological changes they have gone through, have become more balanced. As a result of their maturity, they have developed the "ability to control... emotions and modulate... behavior appropriately (17)." Despite challenges in life, mature brains have the "capacity to ride out emotional storms [with] more flexibility and resiliency (17)." Cohen (2005) also mentioned a research conducted by Canli and his colleagues at the University of California, Santa Cruz, about the nature of older adults. Among their findings were: As adults matured, their experience of negative emotions became less severe; older adults paid more attention to positive stimuli rather than negative ones; elders were more likely to remember positive experiences rather than those which were negative. They concluded that as an individual matured, his "amygdalae showed decreased reactivity to negative information while maintaining or increasing their reactivity to positive information (18)." As a result, older adults became generally "calmer in the face of life's challenges." Amygdala is part of the brain that is involved with processing emotions.

In Del Villar's 2014 study about the correlation between self-worth and selected communication traits among Filipino elders, it was revealed that majority of the respondents had a high positive attitude about aging. This means that, on the average, the aging respondents viewed aging as good, right, beneficial, fair, and positive. Only $4.4 \%$ of the respondents saw aging as bad, wrong, harmful, unfair, and negative.

Based on these previous findings, it can be inferred that attitude about aging is an essential ingredient to successful aging.

\section{$\underline{\text { Pro-active and Meaning-based Acceptance Coping Strategies }}$}

Holahan \& Moos' (1987) argued that, among the coping strategies, two 
appeared to be positively related to successful aging. First was a strategy that allows the elders to be pro-active in finding solutions to the challenges related to their aging. They take an active role in planning their lives using the available resources they probably have previously planned and worked for. This is about focusing on something constructive so that they may be the best they can possibly be. This is also about making plans to improve their situation. Second was a strategy that permits a positive and meaning-based acceptance of their aging. This allows the elders to seek the appropriate philosophy that will help them understand and positively accept the realities of aging. This is about accepting the realities of aging even as they find joy and fulfillment. More importantly, this is about seeing aging as a natural process and the culmination of one's life mission.

In the Del Villar definition of successful aging, the two coping strategies (pro-active and meaning-based acceptance of aging strategies) were included among the important ingredients that make up successful aging.

\section{$\underline{\text { Self-esteem }}$}

Wrench, McCrosky, \& Richmond (2008) defined self-esteem as the "view people have of themselves in terms of total worth (48)." Those who have low self-esteem "tend to lack confidence in their own ability and to evaluate their own competence negatively...They expect failure in whatever they attempt to accomplish, including interactions with others (48)."

Rosenberg (1965) earlier indicated that people with high-self esteem are satisfied with themselves, feel that they "have a number of good qualities," "able to do things as well as most other people," "have so much to be proud of," "feel that [they] are persons of worth," respect themselves, and have a positive attitude towards themselves.

As illustrated by McKay \& Fanning (1992) self-esteem has been repeatedly proven in the field of social sciences as vital to human survival. Without it, one's existence could be "enormously painful (54)." They stressed that one mark that distinguished humans from animals was having the ability to be aware of oneself and attaching value to it. The problem with this, they emphasized, was that when you have a low valuation of yourself (for whatever reason) you also "reject parts of yourself... [thus damaging] the psychological structures that literally keep you alive (1)." This self-rejection could result in the individual avoiding any perceived source of the problem. For instance, the person might avoid any forms of interactions with other people because they might bring him pain. The person could literally limit his "ability to open [himself] with others, express sexuality, be the center of attention, hear criticism, ask for help, or solve problems." The problem could become worse if the person builds boundaries to protect himself. In examining the causes and effects of self-esteem, McKay \& Fanning (1992) pointed out the difficulty saying that the issue could be compared to the "chicken and egg' question. As they analyzed, "it seems that self-esteem grows out of your circumstances in life, and at the same time, your circumstances in life are influenced strongly by your self-esteem. Which came first? (54)." 
Whichever came first, or whichever was the cause or the effect, self-esteem or the circumstances surrounding people, their self-esteem would be extremely important because it would affect their whole being. Having a positive wellbeing would always be associated with feelings of self-confidence. And if people were confident about themselves, they would also be at ease whatever their age. If they were confident they would also become more willing and consequently satisfied with communicating with people around them. If there was one variable that could be used to gauge people's general well-being, selfesteem should be it.

Based on studies about self-esteem, it was hypothesized that self-esteem and the circumstances surrounding the elders would be positively correlated. The better the elders' self-esteem, the better their circumstances. Or, as McKay and Fanning (1992) confounded, the better the circumstances surrounding the elders, the better their self-esteem.

In Del Villar's (2014) study correlating the Filipino elders' self-worth with selected communication traits, it was revealed that majority of the respondents (455 or $57.2 \%$ ) registered high self-esteem, 300 or $37.7 \%$ rated moderate, while 40 or $5 \%$ rated low self-esteem. The Mean self-esteem score was $36.81(\mathrm{SD}=7.42)$ which meant that on the average respondents had a high level of self-esteem. Based on the indicators of self-esteem (Rosenberg, 1965), the elders generally felt satisfied with themselves, thought they were good most of the time, felt that they had "a number of good qualities," were able to do things "as well as most people," did not feel useless, felt that they were persons of "worth when compared with others,' had respect for themselves, did not feel they were a failure, and had a 'positive attitude toward themselves.'

From these findings, it could be inferred that a positive self-esteem was an important ingredient of successful aging.

\section{Happiness}

Helliwell et al. (2013) defined happiness in two ways - "first as an emotion (Were you happy yesterday?) and the second as an evaluation (Are you happy with your life as a whole?)." In their worldwide survey, a clear distinction was made between the two: Happiness as an emotion and happiness with life as a whole or "sense of life satisfaction." In their yearly surveys comparing the happiness index of countries around the globe, three types of measures were used: Measure of positive emotions that included how the respondents felt the day prior to the survey, measures of negative emotions that included how negatively the respondents felt the day prior to answering the survey, and their evaluations of life as a whole. These three types of measures were referred to as the "primary measures of subjective well-being."

Oishi et al. (2013) argued that happiness has been referred to as subjective well-being (SWB) in research in the last 30 years and that the real meaning of the concept has been "elusive." It was precisely for this reason that Diener (1984) advocated the use of the term subjective well-being or SWB instead of happiness because of the uncertainties that have been associated with the term. 
As the term SWB came to be more popularly used to refer to "subjective evaluation of life as a whole, the presence of pleasant emotions, and the relative absence of unpleasant emotions" (542) in the field of psychology, the term happiness was likewise more often used thus leading back to the previous problem of its meaning. So, Oishi et al. (2013) sought to trace the historical and cultural connotations of the term. Their findings showed that the ancient meanings of happiness "centered around good luck, fortune, or external conditions in general" and that these were mostly used in collectivistic cultures. The connotation, however, was not the same in individualistic cultures. More evidence showed that culture played an important role in conceptualizing the term happiness with many cultures still focusing on fortune and luck. There were the exception of some cultures like that of the Americans which saw happiness as "feelingcentric," attainable, and conscious.

Sheehy (1995) stated that elders, women especially, actually became happier as they grew older. She stated that "clinical disorders, including depression, continue to drop off in older women. The peak age for depression has now descended into the mid thirties (192)." The first findings about this improvement in the happiness of elder women came out from the studies by Strole and Fischer (1980 as cited in Sheehy 1995) when he did a follow up of the 1954 Midtown Manhattan Study. He discovered that women "enjoyed marked progress in mental health...The improvement in mental health and well-being among older women was accumulating generationally (193)." Similarly, a report from Millman, an associate of Strole (Sheehy 1995) found a strong correlation between happiness and age also among women. Women became happier as they aged.

Men too became happier as they aged. According to Vaillant (1990 in Sheehy 1995), by the time they reached 65 they have developed "self-mastery." They have acquired the "ability to handle life's accidents and conflicts without passivity, blaming, or bitterness (356)". Overall, traits that contributed to happiness have shifted from "spontaneity, creative flair, and the knack of easy sociability to... being dependable, well organized, and pragmatic (356)."

From these findings, it can be inferred that happiness, which refers to a positive, bright, buoyant general evaluation of life as a whole, is an important ingredient of successful aging.

\section{Successful Aging and Selected Communication-Demographic Factors}

A number of past researches have identified certain communicationdemographic factors as being associated to the elders' successful aging. The following section reviews those past researches.

In a study by Momtaz et al. (2011), age, sex, marital status, and income were found to be significant predictors of the well-being of Malaysian elders. They further found that those who were older, single, and poor were the ones more likely to experience low well-being.

Nosraty et al. (2012) likewise found that men (rather than women) living in a community were more likely to experience successful aging. In addition, younger elders who were married, and had higher education were also more 
likely to experience successful aging. The very old were also likely to experience successful aging if they were independent, adaptable, and had a clear sense of purpose.

Contrary to the above findings, McLaughlin, Connell, Heeringa, and Robert (2009) found that very few Americans were aging successfully. They gave the figure that no greater than $11.9 \%$ were experiencing successful aging. The odds of successful aging were lower for those who were older, male, and lower in the socioeconomic class. Very few older adults met the three criteria previously forwarded by Rowe and Kahn (1997) about successful aging which were "low probability of disease and disease-related disability, high cognitive and physical functional capacity, and active engagement with life (433)."

Similarly, Strawbridge et al. (2002) found that the odds of experiencing successful aging decreased by as much as $25 \%, 18.5 \%$, and $11.6 \%$ among elders in the age brackets 65 to 69,70 to 79 , and 80 to 99 respectively. In the same light, Depp and Jeste (2009) and Depp et al. (2007) revealed that the most consistent predictor of successful aging was being closer to age 60 .

In Braveman et al.'s study (2005), it was found that the prevalence of successful aging was more likely for those belonging to higher socio-economic status and that related indicators such as education, income and wealth were also significantly associated.

On the topic of elders' ability to normally function in daily activities (functionality), and their connection with friends and family (social contact), Pavela's (2015) study discovered that elders who had wider social contact with friends and family had higher levels of functionality. This longitudinal study which found significant correlation between social contact and functionality had a large sample size of over nine thousand elders living outside institutions whose ages were 70 and over.

In 2015, Collinet's paper emphasized the importance given to physical activities and how that importance influenced public policy on health among the aging population.

In a study that aimed to discover predictors of engagement among 10,764 French elder males aged 60-74, Sabbath et al (2015) found that men were more engaging in community volunteerism than women, that women were stronger in care giving and social interaction, that men and those belonging to higher socio-economic status tended to participate in activities than women or those belonging to lower socio-economic status.

Curtis' study in 2015 discovered a link between Australian elders' perception of the quality of control they have over their lives and their general ability to function. Likewise, their ability to function was also linked to the quality of their social activities. Those with poorer perception of control experienced decline in social activities over time. Curtis' study included 835 participants between the ages of 69 to 103 .

Zhang, Feng, Liu, and Zehn (2015) studied the important factors that influenced Chinese elders and discovered that finances, health, living conditions, community activities, and caring for grandchildren were the most influential. Community activities were found to be more important to the elderly women 
while caring for grandchildren was more important to elderly men.

With the objective of identifying subgroups of centenarians with common characteristics that predicted successful aging, Araujo, Ribeiro, Teixera, and Paul (2015) conducted a study involving 80 centenarians in a face to face interview. Results revealed two significant predictors of successful aging: being male and having adequate income.

In an attempt to find the relationship between social engagement and selected demographic variables among elder Chinese living in the US, Dong, Li, and Simon (2015) conducted a study and discovered that as respondents got older, their experience of social engagement became less. Further, the more positively the elders' perceived their health and quality of life, the more they experienced and engaged socially. In addition, contrary to previous findings, their study found that income was not significantly related to elders' social engagement.

In a longitudinal study of 2,032 elders which aimed to find out the benefits of having friends among the elderly, the 2014 study conducted by Huxhold et al. found that activities with friends increased positive affect and life satisfaction and decreased negative affect. These results suggested that social activities with friends became more important and may even have a shock absorbing effect against the negative consequences of aging.

Xu et al. (1999), Shi (1985), and Hu (1985) agreed that the elders' health as well as standing in the family were highly influential in the elders' feelings of well-being. Other factors were income, marital status, educational level, and number of children.

$\mathrm{Xu}$, and Wang, (1994), and $\mathrm{Xu}$, Jiang, and $\mathrm{Wu}$, (1992) found that daily activities and marriage significantly influenced the elders' mental health, quality of life, and well-being. Other factors that were also found to be important were recreation such as dancing and volunteering.

Meng, (1991) found that living with the family directly influenced the elders' quality of life.

Li, Li, \& Chen, (1991) and Shi, \& Ren, (1988) likewise found that elders who lived satisfactorily with their family and had good marriage experienced better mental health.

Wood, \& Roberson, (1976) discovered that those who were involved in grandparenting were the ones who experienced more life satisfaction. Kivnick, (1980) too found connection between grandparenting and life satisfaction. From his study, five dimensions of the meaning of grandparenting emerged: As a "valued elder," "Immortality through clan," "responsible for family well-being," "re-involvement with the personal past," and "indulgence." 


\section{Hypothesis}

From the above discussions about communication-demographic variables and their varying associations to successful aging, it is hypothesized that the selected twenty (20) communication-demographic factors of the present study are linked (positively or negatively) to the Filipino elders' successful aging.

\section{Method}

The study employed both quantitative and qualitative techniques with survey as the main method. The same sample of 816 elders (60 years old or older, willing and able to participate) used in the 2015 study were again used in the present study. Respondents were purposively taken from different sectors of society namely: Government and private institutions for the elderly catering to the low, middle, and upper socio-economic classes, elders living independently, and those living with their families.

\section{Instruments}

All instruments used in the study were based on the indicators established by previous studies and modified to adapt to the elder respondents. Their reliability ratings were tested in the previous study. These are:

1. Coping Strategies (Del Villar 2013) (modified from Carver et al. 1989).

2. Happiness Scale (Del Villar 2013)

3. Attitude about Aging scale (modified from Wrench et al. 2008)

4. Self-esteem Scale (modified from Rosenberg 1965)

Data Analyses

To determine if successful aging was related to communication-demographic factors, the scores in each of the 20 factors were correlated with the successful aging score using the Gamma correlation test at alpha .05.

\section{Results}

\section{Demographic Profile of the Respondents}

There were 816 respondents who participated in this study. Ages ranged from 60 to over 95 where majority $(373,46.2 \%)$ belonged to the 60 to 65 age bracket followed by $188(23.3 \%)$ from the 66 to 70 bracket, $107(13.3 \%)$ from the 71 to 75 group, $67(8.3 \%)$ from the 76 to 80 group, $41(5.1 \%)$ from the 81 to 85 group, $18(2.2 \%)$ from the 86 to 90 group, $11(1.4 \%)$ from the 91 to 95 age group, and $2(.2 \%)$ from those over 95 years old. Four hundred forty nine 
$(449,55.2 \%)$ were females while $364(44.7 \%)$ were males.

Majority or $502(61.6 \%)$ were still working while $310(38 \%)$ were no longer employed. Majority $(601,73.7 \%)$ belonged to the middle class, $151(18.5 \%)$ to the lower class, and only 57 (7\%) to the upper class.

Majority $(503,61.6 \%)$ owned houses and lived with family or relatives, $181(22.2 \%)$ lived in houses owned by family or relatives, $101(12.4 \%)$ lived alone in their own houses, and $29(3.6 \%)$ lived in private or government institutions for the aged.

Majority (452, 55.3\%) was married, $232(28.4 \%)$ were widowed, $85(10.4 \%)$ were single, $45(5.5 \%)$ were separated, divorced or annulled.

Majority $(279,34.2 \%)$ finished college while $90(11 \%)$ pursued graduate education. One hundred thirty two $(132,16.2 \%)$ had partial college education, 170 (20.8\%) had high school or partial high school education, $136(16.7 \%)$ had elementary or partial elementary education. Only 7 indicated no form of education.

Eighty eight percent $(88.7 \%, 724)$ have stopped pursuing continuing education or training while $88(10.8 \%)$ still pursued some forms of education.

Majority of the elders or $558(68.4 \%)$ engaged in some forms of leisure while $253(31 \%)$ had none whatsoever.

One hundred three $(103,12.6 \%)$ experienced excellent health, $518(63.5 \%)$ were in good health, $167(20.5 \%)$ were in a not "so good" health, while only 24 (2.9\%) were in bad health. Among the elders, 290 (35.5\%) exercised regularly, $312(38.2 \%)$ exercised occasionally, and $209(25.6 \%)$ did not exercise at all.

\section{Successful Aging}

The Mean successful aging score of the elder respondents was high at 13.43 ( $\mathrm{SD}=1.72$ ) which means that they generally enjoyed successful aging. Scores ranged from 3 (lowest) to 16 (highest). Majority $(678,83.1 \%)$ rated high, $117(14.3 \%)$ rated moderate and only $1(.2 \%)$ rated low.

Table 1 below shows the distribution of successful aging scores:

Table 1. Successful Aging Score Distribution

\begin{tabular}{|l|c|c|}
\hline & Frequency & Percent \\
\hline High $(11.7-16)$ & 678 & 83.1 \\
\hline Moderate $(7.4-\mathrm{f} 11.6))$ & 117 & 14.3 \\
\hline Low $(3-7.3)$ & 2 & 2 \\
\hline Total & 797 & 100 \\
\hline
\end{tabular}

It is to be recalled that, in the present study, successful aging has four components: Positive attitude about aging, use of coping strategies A and B, high level of happiness, and positive self-esteem. This is based on the assumption that if an elder experiences a positive attitude about aging, is proactive in dealing with the challenges of aging, derives fulfilling meanings in aging, is generally happy, and has a positive self-esteem; he or she also 
experiences successful aging.

The following sections discuss the results of each of the four elements of successful aging. It is to be noted that scores in these four elements were combined to make up the successful aging score.

\section{Attitude about Aging}

The respondents in the study had a Mean attitude about aging score of $23.01(\mathrm{SD}=4.49)$ which corresponds to a very high positive attitude. Majority or $645(79 \%)$ rated very high in attitude, $154(18.9 \%)$ rated moderate, and 9 $(1.1 \%)$ rated low. The elder respondents felt that aging was good, right, fair, and wise because:

- They have the privilege to see their family grow.

- They participate in the normal process of life, the natural order of things.

- They become the younger generation's role model.

- They gain a sense of accomplishment.

- They get the chance to realize the true meaning of life.

- They get to experience the cycle of life - birth and death.

- Their experiences help them gain wisdom.

This was in line with what Cohen (2005) argued: Older adults in general possessed a positive attitude and this was more of a rule than an exception. Among the reasons he gave were the elders' wide understanding and acceptance of life's realities making it easy for them to take on the difficulties that came their way. In addition, because of the physiological changes in the older brains, elders became more emotionally stable and in control. The present study's findings also supported Del Villar's 2014 study which discovered that older adults had a high positive attitude about aging.

There were also some who had a negative attitude about aging. Some elders felt that aging was bad, wrong, unfair, and unwise because:

- Aging makes you weaker.

- Aging causes your looks to fade.

- You become prone to sickness and your mental faculties deteriorate.

\section{Coping Strategy}

A total of 222 (27.2\%) elders chose coping strategy A (pro-active) and 344 (42.2\%) preferred strategy B (meaning-based acceptance) when dealing with the challenges of aging. Scores in each of these two strategies range from 2 (lowest) to 10 (highest). The Mean score in strategy A was 8.4 while in B was 8.8. It is to be noted that respondents were allowed to rate as many strategies as they preferred. Although the pro-active and meaning-based strategies were not the highest ranking choices, they were nonetheless chosen by a sizable number of elders when they had to find ways of dealing with the challenges of life. 
However, only strategies A and B were given points because they were the only two strategies included among the elements of successful aging. The most often used strategy among the respondents was strategy $\mathrm{C}$ (religion) which was chosen by $481(58.9 \%)$. The least used strategy was strategy $\mathrm{F}$ or denial and non-coping $(32,3.9 \%)$.

As Holahan \& Moos (1987) theorized, coping strategies A and B were the ones positively related to successful aging. Strategy A calls for elders to be proactive in finding solutions to the problems of aging. This strategy allows elders to be actively involved in improving their lives using the resources they have. Strategy B calls for elders to have a positive and meaning-based acceptance of their aging. This allows them to seek the most appropriate philosophies in understanding and positively accepting the realities of aging.

In the FGDs, a number of elders disclosed that they have started seeing aging as a natural process of life. This was not their belief when they were younger but age somehow made them accept aging as inevitability. Having this outlook offered a more peaceful view of life. Some saw aging as a culmination of their life purpose. They may not have seen it right away, but aging made them realize what their purpose was and that made it more acceptable. But having a meaningful understanding of aging was not enough. Some made conscious efforts to improve their condition whatever resources they had at their age. Some elder respondents admitted learning new skills to help improve their financial status. One 65 year old retired employee, for instance, enrolled in a wealth management seminar and seriously considered starting a small business that would allow her money to grow. Another invested in medium risk investments in her bank.

\section{Self-esteem}

In self-esteem, the Mean score was $21.02(\mathrm{SD}=3.2)$ which means that on the average, the elder respondents had high self-esteem. Scores ranged from 5 (lowest) to 25 (highest). Majority or 655 (80.3\%) rated high, $150(18.4 \%)$ rated moderate, while $5(6 \%)$ rated low. This means that generally, elders felt satisfied with themselves, thought they were good most of the time, believed that they had some good qualities to be proud of, accomplished important tasks as well as others, never felt useless, thought they were persons of worth, had respect for themselves, never thought of themselves as a failure, and had a general positive attitude about themselves.

During an FGD, a number of elders who rated high in self-esteem illustrated that they had the capacity to lead active lives. Although they were mostly retired from employment, they still found a number of reasons to be active, involved, and useful in their families or even communities. According to them, they were probably still alive because they were not yet done with their life mission.

This result agreed with the previous study by Del Villar (2014) where majority of Filipino elders (455 or 57\%) registered high self-esteem, 300 or $37.7 \%$ rated moderate, while only 40 or $5 \%$ rated low. 


\section{Happiness}

The happiness Mean score was 5.89 ( $\mathrm{SD}=1.09)$ which means that, on the average, the elder respondents were happy. The highest possible score was 7 for very happy and the lowest was 1 for very unhappy. The biggest percentage (35.8\%) or 292 rated 7 (very happy), followed by $259(31.7 \%)$ who rated 6 (happy), $169(20.7 \%)$ rated 5 (moderately happy), $66(8.1 \%)$ rated 4 (neutral), $17(2.1 \%)$ rated 3 (moderately unhappy), $4(.5 \%)$ rated 2 (unhappy), and only 1 $(.1 \%)$ rated very unhappy.

During the FGDs, a number of respondents disclosed that compared to how they viewed life during their younger years, they were in a much better place now. Although there were still challenges (mainly about health, finances, and family), their perspective have shifted and they were able to see life in a more positive light. They were happier.

\section{Correlations between Communication-Demographic Factors and Successful Aging}

Analyses were done to find out if the 20 selected communicationdemographic characteristics of the elders were related to their successful aging. Results showed that of the 20, eight were not significantly correlated. These were: age, sex, occupation, place of residence, continuing education, mode of communication with close family and friends, and type of religion. The 12 factors that were significantly correlated were: health, civil status, socio-economic status, education, engagement in and frequency of leisure activities, exercise regimen, number of close family, frequency of communication with close family, number of close friends, frequency of communication with close friends, and involvement in religion.

\section{Health}

Results of the Gamma test showed that there was a significant association between the elders' health and their successful aging $(\gamma=.450, p=.000)$. As the respondents rated higher in health, they also rated higher in successful aging. Majority (513 or 64\%) of the elders experienced "good health", followed by 164 (20\%) who experienced "not so good health", then 101 (12.5\%) who admitted feeling excellent health, and only $24(.029 \%)$ who felt they were in "bad health." In the interviews conducted, most of the respondents admitted that if they felt good physically, did not have any serious illnesses, and felt energetic, they also felt happier and more motivated to go about their daily activities. The present findings agreed with the results of a number of other studies namely Zhang et al. (2015), Xu et al. (1999), Shi (1985), and $\mathrm{Hu}$ (1985) which found that health among other factors was influential in the well-being of Chinese elders. Other studies that also agreed with the present findings were Xu et al. (1999), Shi (1985) and $\mathrm{Hu}(1985)$ who all discovered that health was highly influential in the elders' feelings of well-being. 


\section{Civil Status}

When the correlation test was done between civil status and successful aging, results showed that there was a negative correlation between the two $(\gamma=-.190$, $\mathrm{p}=.030$ ). This means that among the respondents, the singles (or those who never married) were the ones who experienced the highest level of successful aging while those who were married, widowed, or separated from their partners experienced less successful aging in comparison. This, of course, does not mean that those who were not single did not experience successful aging because results showed that in general, the elder respondents experienced a high level of successful aging. It was just that the married, separated, and widowed experienced less in comparison to the single respondents. Among the respondents only $84(9.6 \%)$ were single, $444(55 \%)$ were married, 230 (29\%) were widowed, and $45(6 \%)$ were separated from their partners. In the FGD, there were different opinions about the association between civil status and successful aging. A number of married respondents admitted that marriage became more difficult during the later years when their children were no longer with them to provide a strong reason to keep the family together. The presence of the children took much of the focus away from the problems that came with marriage. The single elders, on the other hand, did not have any children and were used to being on their own even during their later years. Some married couples, on the other hand, admitted that being with somebody "through thick and thin" was in itself very rewarding.

Some previous studies on civil status were not supported by the present findings. One such research by Momtaz, et al. (2011), found that being married was related to well-being. This was also in agreement with Nosraty et al' s (2012) study although their respondents were those living together in a community which was different from the present study's respondents who were mostly living in their own residences. Xu, et al. (1999), Shi (1985), and Hu (1985) similarly found that being married was influential in the elders' well-being. Again, one should consider not only the comparison of results among the different civil statuses but the average result of all the elders which was a high rate of successful aging. This means that whatever their status, they generally experience successful aging.

\section{Socio-economic Status}

Results of the correlation test between socio-economic status and successful aging showed a significant positive correlation between the two $(\gamma=.317, \mathrm{p}=.004)$ which means that the higher the socio-economic status, the higher the level of successful aging. Most of the respondents belonged to the middle socio-economic status (444 or 55\%), while only $84(10 \%)$ belonged to the lower socio-economic status and $230(29 \%)$ belonged to the upper socio-economic status. This result made perfect sense because those who were financially capable could enjoy the comforts of life, got the best medical support when needed, and were not burdened by the basic needs of daily life. Momtaz, et al. (2011) also found that income was an indicator of well-being among Malaysian elders. Likewise, 
Braveman et al. (2005) found that income and wealth were significantly related to successful aging among their American respondents. Sabbath, et al.'s (2015) study also discovered that elders coming from higher socio-economic status had more social engagement than their poorer counterparts. Zhang, et al. (2015), too, found that finances were influential in the well-being of Chinese elders. In another study of 80 centenarians, Araujo et al. (2015) confirmed that having adequate income was an important predictor of successful aging. On the contrary, Dong et al. (2015) found that income was not significantly associated with elders' social engagement. Their study was conducted among Chinese living in the US. Xu et al. (1999), Shi (1985), and Hu (1985) similarly found that income was an important factor in the elders' well-being.

\section{Education}

Results showed that there was a significant correlation between education and successful aging $(\gamma=.268, \mathrm{p}=.000)$; the higher the level of education, the better the experience of successful aging. Among the respondents, $273(34 \%)$ had college education, $168(21 \%)$ had partial or complete high school, $136(17 \%)$ had elementary or partial elementary education, $131(16 \%)$ had partial college, and only $87(11 \%)$ had graduate level education. In an interview of respondents with a graduate education, they acknowledged that their education must have helped them deal with their aging in a mature way. Similarly, those who finished a college degree felt the same way. They felt more open and sophisticated in facing the challenges of aging. One respondent for instance revealed that he felt that he was more ready to plan for his old age because of the education he received during his younger age. This result was in agreement with what Nostrasty et al. concluded in 2012 wherein their educated elder respondents experienced a higher level of wellbeing. In the same light, Braveman et al. (2005) found education to be associated to the well-being of American elders. Xu et al. (1999), Shi (1985), and Hu (1985) similarly agreed that the elders' educational level was positively linked to their well-being.

\section{Engagement in Leisure}

Results of the correlation test showed that there was a positive link between level of engagement in leisure activities and successful aging $(\gamma=.427, \mathrm{p}=.000)$. Elders who engaged in more activities experienced a higher level of successful aging than those who had fewer activities. Among the respondents, 51 (69\%) engaged in moderate levels of activities, $250(31 \%)$ in high levels of activities, and only 1 engaged in very low level of activities. One respondent admitted that keeping herself busy with her craft made her excited about facing each day. What started as a hobby now provided her with additional income. What is more, receiving compliments for her nice craftsmanship gave her something to be proud of. Another elder declared that playing leisurely golf with his friends every Wednesday gave him something to be excited about. Zhang et al. (2015) too found that community activities were influential in the well-being of Chinese 
elders. Xu et al. (1994) and Xu et al. (1992) similarly found that daily activities significantly influenced the elders' mental health, quality of life, and wellbeing. Other factors that were also found to be important were recreation such as dancing.

\section{Frequency of Leisure}

Not only was engagement in leisure activities good for successful aging. the frequency of engaging in similar activities was also beneficial. Test results showed that the more frequently elders engaged in leisure activities, the more they experienced successful aging $(\gamma=.273, \mathrm{p}=.002)$. Among the elders, $310(39 \%)$ engaged in leisure activities regularly, 266 (33\%) engaged occasionally, and 223 $(28 \%)$ never engaged in leisure activities. In a focus group discussion, members of a community ballroom dance admitted that they enjoyed dance times with their friends. They met on Tuesdays at the village clubhouse and with a dance instructor leading the dances; they happily danced for an hour or two.

\section{Exercise Regimen}

As to exercise regimen, correlation results showed that the more the elders exercised on a regular basis, the more they experienced successful aging $(\gamma=.211$, $\mathrm{p}=.015)$. Among the respondents, 310 (39\%) exercised occasionally, $285(35 \%)$ exercised regularly, while 206 (26\%) did not exercise at all. One male respondent said that brisk walking around his subdivision for 30 minutes every afternoon was enough exercise for him. Rain or shine, he would walk. At times, he would force himself to do his daily rounds even if he was feeling lazy. He claimed that this daily routine seemed to improve his overall feeling of well being. Another elder woman admitted to just staying home and watching her daily soap opera on television. Doing any kind of exercise did not appeal to her because her knees were already badly swollen with arthritis. She also admitted that lack of exercise was probably the cause of her insomnia which in turn gave her the bad mood on most days.

\section{Number of Close Family}

In the Filipino family, having a close relationship with each other was important and contributed to an elder's successful aging. This was confirmed in the correlation test between number of close family members and successful aging $(\gamma=.156, p=.022)$. The bigger the number of close family members the elders reported, the higher their level of successful aging. A number of elder respondents happily admitted that being surrounded by their adult children and grandchildren made them feel secure. However, this was not the same case with everybody as some elders who lived alone felt comfortable even without a companion. According to one, there was joy in living in solitude. The present study's findings supported that of Pavela's research (2015) wherein he found that wider social contact with family improved the elders' level of functionality. His study involved 
a large sample size of over nine thousand elders living outside institutions. Zhang et al.'s (2015) study showing that having a close relationship with family, specifically caring for grandchildren, was also confirmed to have a positive influence on successful aging. Other studies (Meng 1991, Li et al. 1991, Wood et al. 1996) were also supported by the present findings that elder respondents who had a close relationship with family also experienced successful aging.

\section{Frequency of Communication with Close Family}

In addition to number of close family members, frequency of communication with them was also important. Results of the correlation test showed that the higher the frequency of communication with family members, the higher the level of successful aging $(\gamma=.346, p=.004)$. Among the elders, $642(80 \%)$ had regular contact with close family member, $126(16 \%)$ had occasional contact, 18 $(2 \%)$ and $16(1 \%)$ had never or only yearly contact with close family respectively. The other factor, mode of communication, did not significantly correlate with successful aging. This means that the manner of communication was not as important as the mere presence and frequency of communication with family members.

\section{Number of Close Friends}

As to number of close friends, results showed that the more close friends the elders reported, the higher their level of successful aging $(\gamma=.132, \mathrm{p}=.049)$. The elder respondents felt happier when with friends. This result confirmed Pavela's (2015) study which discovered that elders who had wider social contact with friends and family had higher levels of functionality. In a longitudinal study of a large group of elders, Huxhold et al. (2014) confirmed that activities with friends increased life satisfaction and decreased the negative effects of aging.

\section{Frequency of Communication with Close Friends}

As to frequency of communication with their close friends, elders reported having higher level of successful aging as their number of close friends increased $(\gamma=.186, p=.05)$. The manner by which they communicated, either by face to face or by use of other means, was not as important as the frequency of having contact with them.

\section{Involvement in Religion}

Finally, another significant link to successful aging was the elders' involvement in their religion $(\gamma=.362, p=.000)$. The more active they were in the practice of their religion, the higher they rated in successful aging. Of all the respondents, $366(46 \%)$ rated high level of involvement, $249(31 \%)$ rated moderate, and 185 (23\%) rated low participation. Nobody rated no participation. The type of religion did not matter significantly to the elders. What was important 
was the quality of involvement they had. In one observation conducted in a church choir composed of elder women, they were in high spirits whenever they practiced their songs together. They would cheerfully rehearse once a week, and sometimes more often if they thought there was a need to learn new songs.

The table below shows the summary of the 12 significant correlations between successful aging and communication-demographic factors:

Table 2. Summary of Significant Correlations between Successful Aging and the 12 Communication-Demographic Variables

\begin{tabular}{|l|c|}
\hline Gamma Correlation & Successful Aging \\
\hline Health & $\gamma=.450, \mathrm{p}=.000$ \\
\hline Civil Status & $\gamma=-.190, \mathrm{p}=.030$ \\
\hline Socio-economic status & $\gamma=.317, \mathrm{p}=.004$ \\
\hline Education & $\gamma=.268, \mathrm{p}=.000$ \\
\hline Leisure activities & $\gamma=.427, \mathrm{p}=.000$ \\
\hline Frequency of leisure activities & $\gamma=.273, \mathrm{p}=.002$ \\
\hline Exercise regimen & $\gamma=.211, \mathrm{p}=.015$ \\
\hline Number of close family & $\gamma=.156, \mathrm{p}=.022$ \\
\hline Frequency of communication with close family & $\gamma=.346, \mathrm{p}=.004$ \\
\hline Number of close friends & $\gamma=.132, \mathrm{p}=.049$ \\
\hline Frequency of communication with close friends & $\gamma=.186, \mathrm{p}=.052$ \\
\hline Involvement in religion & $\gamma=.362, \mathrm{p}=.000$ \\
\hline
\end{tabular}

Overall, the findings seem to suggest that being healthy, being single (more than being married or separated or divorced), being financially capable, being educated, frequently indulging in leisure activities, having an exercise regimen, being frequently close to more family members and friends, and being actively involved in religious activities are significantly linked to the successful aging of Filipino elders.

\section{Summary and Conclusion}

This study is an extension of a previous research significantly correlating Filipinos' successful aging to selected cultural orientations. The same 816 elder respondents' communication-demographic characteristics were further evaluated to determine their link to successful aging.

As test results showed, 12 of the 20 selected communication and demographic factors significantly correlated with successful aging. These were health, civil status, socio-economic status, education, engagement in and frequency of leisure activities, engaging in an exercise regimen, number of close family, frequency of communication with close family, number of close friends, frequency of communication with close friends, and involvement in religion. The other eight (8) factors were not significant. These were: age, sex, occupation, place of residence, continuing education, mode of communication with close family and 
friends, and religion.

This study suggests that, in addition to cultural orientations (Del Villar 2015), certain communication-demographic factors are also linked to the Filipino elders' successful aging. This confirms what previous studies have discovered about elders in other parts of the world. It should be noted that there were also contradictions regarding certain findings. To name one, being married was not found to be the top ranking link to the Filipino elders' successful aging. This did not support previous research findings, where being married was a high ranking indicator of well-being. Instead, it was being single that was the most prominent. It should however be noted that being married was also correlated with successful aging. It was just that, in comparing the different civil statuses, it was being single that rated the highest level of successful aging. Other factors found to be important to successful aging in past studies but were not found to be significant in the present study were age, sex, occupation, place of residence, continuing education, mode of communication with close family and friends, and type of religion.

On the whole, one can infer from the findings that Filipino elders experience successful aging if they were in good health, single, of good financial standing, educated, actively involved in leisure and exercise activities, with many close family and friends who communicate with them regularly, and involved in religious activities.

A practical implication of the present findings is in the area of policy recommendations for government or private institutions concerned with the welfare of the aging population. For instance, the Department of Health could initiate more programs that could help enhance good health among the elderly; Community based financial or business organizations could organize trainings to help elders improve their financial capabilities in terms of managing their existing wealth or even earning money; The Department of Education could include some kind of regular training workshops or even formal adult education for elders who wish to continue learning; City governments could set-up more leisure and exercise programs for the elders; Social groups could sponsor information drives about the importance of family and social interactions with the elders; Lastly, the church could organize religious activities that involve the elders of the community.

Another important implication of the study is that the Filipino elders' successful aging is so complex and is probably also linked to a hundred more other factors. Of those possible factors, only 20 were tested in the present study. And as shown by the analyses, some concurred with previous findings while others did not thus stressing the need to conduct further investigations until more questions are satisfactorily answered.

In the future, more attention could be given to the way successful aging is defined by different studies. Identifying common elements among those definitions could serve as the basis for making more credible comparisons among results of different studies.

One thing is certain though. This study is an addition to the growing number of literature about Filipino elders. And because some questions were raised as a 
result, the process of finding answers to those new questions continues.

\section{References}

Araujo L, Ribeiro O, Teixeira L, Paul C (2015) Predicting Successful Aging at One Hundred Years of Age. Research on Aging 38(6): 689-709.

Braveman PA, Cubbin C, Egerter S, Chideya S, Marchi KS, Metzler M, Posner S (2005) Socio-economic status in health research: One size does not fit all. JAMA 294(22): $2879-88$.

Carver C, Scheier M, Weintraub J (1989) Assessing coping strategies: A theoretically based approach. Journal of Personality and Social Psychology 56: 267-283.

Cohen G (2005) The mature mind: The positive power of the aging brain. New York: Basic Books.

Collinet C, Delalandre M (2015) Physical and sports activities, and healthy and active ageing: Establishing a frame of reference for public action. International Review for the Sociology of Sport 52(5): 570-583.

Curtis R, Windsor TD, Luszcz MA (2015) Perceived control moderates the effects of functional limitation on older adults' social activity: Findings from the Australian longitudinal study of ageing. The Journals of Gerontology Series B 72(4): 571-581.

Del Villar C (2015) Correlation between cultural orientations and successful aging of Filipinos. Journal of Media and Communication Studies 7(7): 122-145.

Del Villar C (2014) Correlations among attitude about aging, willingness to Communicate, communication satisfaction, and self-esteem of Filipino elders. Journal of Media and Communication Studies 6(9): 150-160.

Del Villar C (2013) Correlation between Compliance-Gaining Strategies and High- Power Distance Cultural Orientation of Filipinos: Re-Validation. Human Communication 16 (3): 133-152.

Depp C, Jeste D (2009) Definitions and predictors of successful aging: A comprehensive review of larger quantitative studies. FOCUS 7(1): 137-50.

Depp C, Glatt S, Jeste D (2007) Recent advances in research on successful or healthy aging. Current Psychiatry Reports 9(1): 7-13.

Diener E (1984) Subjective well-being. Psychological Bulletin. 95: 542-575.

Dong X, Li Y, Simon M (2015) Social Engagement Among U.S. Chinese Older Adults Findings From the PINE Study. The Journals of Gerontology Series A 68(2): 582589.

Elejalde-Ruiz A (2011) How old do you feel inside: The key to staying healthy and living longer is deciding you're not old and decrepit. Lifestyles Chicago Tribune.

Heckhausen J, Krueger, J (1993) Developmental expectations for the self and most other people: Age grading in three functions of social comparisons. Developmental Psychology. 29, 539-548. In T Hess (2006) Handbook of the Psychology of Aging. Massachusetts, California, London: Academic Press.

Heckhausen J, Brim O (1997) Perceived problems for self and others: Self-Protection by social downgrading throughout adulthood. Psychology of Aging 12(4): 610-9.

Helliwell J, Layard R, Sachs J (2013) World Happiness Report 2013. Agency of the United Nations.

Holahan C, Moos R (1987) Risk, resistance, and psychological distress: A longitudinal analysis with adults and children. Journal of Abnormal Psychology 96: 3-13.

Hu J (1985) Preliminary analysis of the factors affecting life satisfaction in retired aged people. Journal of Gerontology, 4, 18-21. In W Zhenyun (1996) The Psychology of 
Aging in China. World Psychology 2(1): 71-78. [in Chinese].

Huxhold O, Miche M, Schuz B (2014) Benefits of having friends in older ages: Differential effects of informal social activities on well-being in middle-aged and older adults. Journals of Gerontology Series: Psychological Sciences 69: 366-375.

Kivnick H (1980) The meaning of grandparenthood. Ann Arbor: UMI Research Press. In A Kornhaber (1996) Contemporary Grand Parenting. Thousand Oaks, California: Sage Publications, Inc.

Levy B (1975) Attitudes towards aging. Journal of Psychological Science. In M Beck (2009) Health matters. The Wall Street Journal (n.d.)

Li S, Li G, Che C (1991) A survey of family, marital, and health status among aged people in an urban area of Beijing. Chinese Journal of Mental Health 5: 203-215. In W Zhenyun (1996) The Psychology of Aging in China. World Psychology 2(1) 71-78. [in Chinese].

McKay M, Fanning P (1992) Self-esteem. The ultimate program for self-help. New York: MJF Books.

McLaughlin S, Connell C, Heeringa S, Li L, Roberts J (2009) Successful Aging in the United States: Prevalence Estimates from a national sample of older adults. The Journals of Gerontology 65B 2: 216-226.

Meng J (1991) The pattern of family and quality of life. Journal of Gerontology 3: 135140. In W Zhenyun (1996) The psychology of aging China. World Psychology 2(1): 71-78. [in Chinese].

Momtaz Y, Hamid TA, Yusoff S, Ibrahim, R, Yahaya N (2011). Loneliness as a risk factor for hypertension in later life. Journal of Aging and Health. 24 (4) 696-710.

Nosraty L, Sarkeala T, Hervonen A, Jylha M. (2012) Is there successful aging for Nonagenarians? The vitality 90+ study. Journal of Aging Research. Article ID 868797. http://dx.doi,org/10.1155/2012/868797.

Oishi S, Graham J, Galinha I (2013) Concepts of happiness across time and cultures. Personality and Social Psychology Bulletin 39(5): 559-577.

Pavela G (2015) Functional status and social contact among older adults. Research on Aging 37(8): 815-836.

Rosenberg M (1965) Society and the adolescent self-image. Princeton, NJ: Princeton University Press.

Rowe J, Kahn R (1997) Successful aging. The Gerontologist 37(4): 433-40.

Sabbath E, Matz-Costa C, Rowe J, Leclerc A, Zins M, Goldberg M, Berkman L (2015) Social predictors of active life engagement: A time-use study of young-old French adults. Research on Aging 38(8): 864-893.

Sheehy G (1995) New passages: Mapping your life across time. New York: Random House.

Shi R, Ren G (1988) The relationship between parents and offspring. Journal of Gerontology 3: 19-142. In W Zhenyun (1996) They psychology of aging in China. World Psychology 2(1): 71-78. [in Chinese].

Shi R (1985) An investigation of some feelings in retired aged people. Journal of Gerontology 1: 40-43. In W Zhenyun (1996) The psychology of aging in China. World Psychology 2(1): 71-78. [in Chinese].

Strawbridge W, Wallhagen M, Cohen R (2002) Successful aging and well-being: Selfrated compared with Rowe and Kahn. Gerontologist 42(6): 727-33.

Strole L, Fischer A (1980) The Midtown Manhattan longitudinal study vs the mental doctrine. Archives of General Psychiatry. In G Sheehy (1995) New passages: Mapping your life across time. New York: Random House.

Vaillant G, Vaillant C (1990) Natural history of male psychological health: A 45 year study of predictors of successful aging at 65. The American Journal of Psychiatry 
147: 31-37. In G Sheehy (1995) New Passages: Mapping Your Life across Time. New York: Random House.

Wood V, Roberson J (1976) The significance of grandparenthood. J Gubrium (ed) Time, roles and self in old age. New York: Human Sciences Press. In A Kornhaber (1996). Contemporary Grandparenting. Thousand Oaks, California: Sage Publications, Inc.

Wrench J, McCroskey J, Richmond V (2008) Human Communication in Everyday Life. Explanations and Applications. Pearson: Boston, New York

Xu S, Wang C, Jiang L, Liu H (1999) The quality of life and subjective well-being of retired professionals and cadres. Chinese Journal of Mental Health 2: 63-66. In W Zhenyun (1996) The psychology of aging China. World Psychology 2(1): 71-78. [in Chinese].

Xu S, Jiang L, Wu Z (1992) The design of the Marriage Satisfaction Inventory and its use among the aged. Chinese Journal of Applied Psychology 3: 29-37. In W Zhenyun (1996) The psychology of aging in China. World Psychology 2(1): 71-78. [in Chinese].

Xu S, Wang C (1994) A preliminary study on the relationship between activity and quality of life as well as subjective well-being in the elderly. Journal of Gerontology 6: 322325. In W Shenyun (1996) The Psychology aging of in China. World Psychology 2(1): 71-78. [in Chinese].

Zhang W, Feng Q, Liu L, Zehn Z (2015) Social Engagement and Health: Findings from the 2013 Survey of the Shanghai Elderly Life and Opinion. International Journal Aging Human Development 80(4): 332-356. 